\title{
An innovative procedure for large-scale synthesis of carbon nanotubes by fluidized bed catalytic vapor deposition technique
}

\begin{abstract}
After the efforts of the first decade, scientists and technicians are facing the big challenge of going from laboratory studies to the large scale production of carbon nanotubes (CNTs) and their ultimate commercial applications. Therefore, innovations in the CNT manufacturing process and its engineering are strongly required. To this contribution, a new technique for the mass production of CNTs by fluidized bed catalytic chemical vapor deposition (FBCVD) has been developed. The CNTs synthesis reactions were carried out in the presence of ironcobalt supported on alumina as a catalyst and ethanol as the source of carbon at $600^{\circ} \mathrm{C}$. The product was characterized using scanning electron microscopy (SEM), transmission electron microscopy (TEM), thermogravimetric analysis (TGA) and X-ray diffractometry (XRD). The results revealed that this technique offers the fabrication of large quantities of CNTs which has the important quality of being free from large amorphous carbon, open-ended with narrow diameter distribution, and having good morphology with few defects. The proposed design has other remarkable advantages, such as simplicity, low cost, energy savings, completely controllable and easy to scale-up, which make it suitable for industrial scale production.
\end{abstract}

Keyword: Carbon nanotubes; Catalytic deposition; Fluidized bed; Large scale-up; Open ended; Synthesis 